\title{
Largest Cross-sectional Diameter at End Ventricular Diastole
}

National Cancer Institute

\section{Source}

National Cancer Institute. Largest Cross-sectional Diameter at End Ventricular Diastole. NCI Thesaurus. Code C127566.

The largest cross sectional diameter measured at end ventricular diastole. 